\title{
Forty-eight new cases with infertility due to balanced chromosomal rearrangements: Detailed molecular cytogenetic analysis of the 90 involved breakpoints
}

\author{
M. MANVELYAN ${ }^{1,2}$, I. SCHREYER ${ }^{2}$, I. HÖLS-HERPERTZ ${ }^{3}$, S. KÖHLER ${ }^{4}$, R. NIEMANN ${ }^{5}$, U. HEHR ${ }^{6}$, \\ B. BELITZ ${ }^{7}$, I. BARTELS ${ }^{8}$, J. GÖTZ ${ }^{9}$, D. HUHLE ${ }^{9}$, M. KOSSAKIEWICZ ${ }^{10}$, H. TITTELBACH $^{10}$,

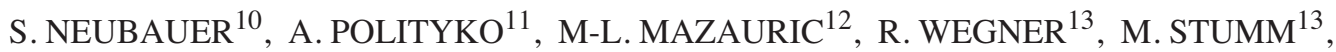

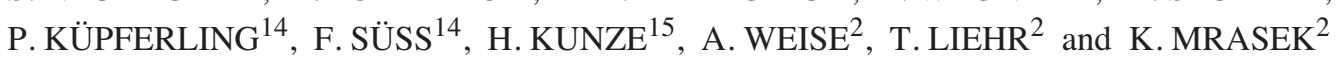

\begin{abstract}
${ }^{1}$ Department of Genetics and Laboratory of Cytogenetics, State University, 1 Alex Manoukian Street, Jerewan 375025, Armenia; ${ }^{2}$ Institute of Human Genetics and Anthropology, Postfach, D-07740 Jena; ${ }^{3}$ Cytogenetic Institute, Vinzenz-Pallotti Street 20-24, D-51429 Bensberg; ${ }^{4}$ Practice of Human Genetics, Frankfurter Street 62, D-97082 Würzburg; ${ }^{5}$ Practice of Human Genetics, Busch 12, D-51491 Overath; ${ }^{6}$ Laboratory of Human Genetics, Hemauerstr. 1, D-93047 Regensburg; ${ }^{7}$ Practice of Human Genetics, Frankfurter Allee 231a (MLZ), D-10365 Berlin; ${ }^{8}$ Institute of Human Genetics, Center for Hygiene and Human Genetics, Heinrich-Düker-Weg 12, D-37073 Göttingen; ${ }^{9}$ Practice of Human Genetics, Postfach 1720, D-5527 Wetzlar; ${ }^{10}$ Practice of Human Genetics, Bankgasse 3, D-90402 Nürnberg, Germany;

${ }^{11}$ Republican Center of Mother and Child, Ministry of Health, Orlovska Street 66, 220053 Minsk, Belarus;

${ }^{12}$ Prenatal Medicine and Genetics, Graf Adolf Street 35, D-40210 Düsseldorf; ${ }^{13}$ Practice of Human Genetics, Kurfürstendamm 199, D-10719 Berlin; ${ }^{14}$ Practice of Human Genetics, Marienstr. 27, D-03046 Cottbus; ${ }^{15}$ Cytogenetic Laboratory, Ahrstr. 2-4, D-45879 Gelsenkirchen, Germany
\end{abstract}

Received January 16, 2007; Accepted February 21, 2007

\begin{abstract}
A molecular cytogenetic study was performed on 48 infertile patients who were identified as carriers of balanced translocations (40 cases), inversions (6 cases) or insertions ( 2 cases) by means of banding cytogenetics. Cases with a Robertsonian translocation or pericentric inversion 2 or 9 were not included. In summary, 100 break-events occurred in these patients, and 90 different chromosomal regions were involved. Thus, this study confirmed the presence of abnormal karyotypes in a subgroup of patients seeking infertility treatment. Breaks were demonstrated to appear preferentially in GTG-light bands in these patients. Furthermore, the observed breakpoints were associated with genomic regions prone to instability due to the presence of segmental duplications. Nonetheless, further detailed molecular
\end{abstract}

Correspondence to: Dr Thomas Liehr, Institut für Humangenetik und Anthropologie, Postfach, D-07740 Jena, Germany

E-mail: i8lith@mti.uni-jena.de

Key words: translocation, inversion, insertion, infertility, breakpoint, fragile site, multicolor banding analysis will be necessary in the future to characterize the mechanisms and genetic basis for this phenomenon.

\section{Introduction}

According to Shah et al (1) infertility is defined as the inability to conceive after one year of regular unprotected intercourse and accounts for one in six couples wishing to start a family. A range of factors may influence fertility. Besides hormone status, age, lack of exercise, obesity or infectious disease infertility factors may be immunological, psychological, resulting from surgery or blockage, or associated with defined abnormalities in the gametes (such as azoospermia). In 20\% of couples the reason for infertility remains unexplained (2). As most, if not all, of the above listed factors are likely to have a genetic component, it is difficult to consider accurately the genetic contribution to reduced fertility. Nevertheless, genetic and/or karyotypic analysis revealed association of specific (cyto)genetic conditions with infertility phenotypes, such as mutations in the cystic fibrosis (CFTR) gene, mutations or microdeletions in $\mathrm{Y}$ chromosome genes, or the presence of constitutional numerical or structural chromosomal aberrations (1). The latter, such as sex-chromosome aberrations, the presence of small supernumerary marker chromosomes (3), constitutional inversions or translocations can lead both to fertility problems and repeated abortions (1). In translocation and in inversion carriers reduced fertility is mediated by the fact that the rearranged chromosomes need to synapse through 
a pairing cross, in order to progress through meiosis. Therefore, in the presence of a rearrangement a multitude of different mechanisms can lead to pairing mistakes during meiosis I or II; only one of these possibilities builds an imbalance to partial trisomy and partial monosomy in regions that are involved in reciprocal translocation or inversion (reviewed in ref. 1). Thus, a balanced chromosomal abnormality in one of the parents is found in up to 5-7\% of couples who experience multiple spontaneous abortions; these patients form a special subgroup within the heterogeneous clinical group 'infertile couples' (4).

To the best of our knowledge, here we present the largest molecular cytogenetic study on otherwise healthy persons with 'uncommon' balanced cytogenetic aberrations detected due to infertility problems. Cases with the more common Robertsonian translocations or pericentric inversions of chromosomes 2 or 9 (5-6) were not included here. Twelve males and 36 females with a family history of repeated abortions and/or a child with congenital malformations, or unexplained infertility were studied by banding and molecular high-resolution cytogenetics. The breakpoints were characterized in detail and are discussed concerning the type of detected rearrangements, distribution of breaks within GTG-light and -dark bands, and the possible correlation of characterized breakpoints and known location of segmental duplications within the human genome.

\section{Materials and methods}

The 48 patients with fertility problems included in this study are summarized in Table I. In 21 cases a history of repeated abortions was observed in partnership, in 4 cases each an unbalanced rearrangement was detected in an unborn fetus or a newborn child with congenital malformations, and in 19 cases there was unexplained infertility. Metaphase chromosome preparations were obtained from PHA-stimulated lymphocyte cultures for each of the studied patients according to standard procedures. Only carriers of 'uncommon' balanced chromosomal rearrangements were included in this study; i.e. no carriers of Robertsonian translocations or pericentric inversions of chromosomes 2 or 9 .

Fluorescence in situ hybridization (FISH) was performed using well-established protocol. High-resolution multicolor banding (MCB) and subcentromere-specific multicolor FISH (subcenM-FISH) were performed as described previously (7-8). Commercially available probes were used according to the manufacturer's instructions (Abbott/Vysis) and are listed in Table I. Ten to twenty metaphase spreads were analyzed, each using a fluorescence microscope (Axioplan 2 mot, Zeiss) equipped with appropriate filter sets to discriminate between a maximum of five fluorochromes and the counterstain DAPI (diaminophenylindol). Image capturing and processing were carried out using an isis mFISH imaging system (MetaSystems, Altlussheim, Germany) for the MCB evaluation.

\section{Results}

The obtained results are listed in Table I. In the 48 reported cases all 24 human chromosomes were involved in the observed rearrangements, each single chromosome at least one time (Fig. 1). In summary, 100 break-events were observed in 90 different chromosomal regions. Five breakpoints were molecularly cytogenetically identical in two cases each, and one breakpoint even in three cases (Table I; Fig. 1). All rearrangements were molecular cytogenetically balanced.

The chromosomal breakpoints were detected in an initial step by GTG-banding. In a second step they were characterized in detail by MCB and, if convenient, additional locusspecific probes were applied (Table I). In summary, only $17 / 100$ breakpoints could be confirmed as determined by GTG-banding alone; 47/100 breakpoints had to be corrected and 36/100 were determined exclusively by MCB; namely, 47 of the 64 breakpoints (73\%) were established incorrectly after GTG-banding alone.

In 38 cases a 'simple' balanced translocation between two chromosomes was present. Two cases each showed either a complex balanced translocation involving three different chromosomes or an insertion of chromosomal material into another chromosome, and in 6 cases an inversion took place.

Evident from Fig. 1 and Table II, 57 of the break-events occurred in GTG-light bands, 3 in hemiheterochromatic regions, 25 in GTG-dark bands and 5 were localized in the transient area of a GTG-dark to a GTG-light band.

\section{Discussion}

One hundred break-events were studied in 48 infertility patients by multicolor banding. One hundred break-events in 48 patients referred for molecular-cytogenetic characterization due to unexplained infertility, repeated abortions and/or born or unborn children with an 'uncommon' chromosomal imbalance are reported here. All patients, except 'unexplained infertility' were clinically normal and not previously reported in the literature; only case 26 was previously described (9). To the best of our knowledge this is the largest such study ever reported.

As previously shown, molecular cytogenetics, especially high-resolution multicolor banding (MCB) (7) enabled a much better characterization of chromosomal breakpoints than GTG-banding. It is striking that $73 \%$ of the GTGbanding-characterized breakpoints had to be reassessed after application of MCB. However, similar data was obtained in previous comparable MCB studies of leukemia and clinical genetics cases (10-12). The high reliability of MCB results has been proven previously by checking all (12) or a subset of the results by other locus-specific probes [(11) and present study, Table I]. Thus, in summary the high rate of GTGbanding results needing revision is alarming and underlines the need for molecular cytogenetic validation. This is preferable in all cases but especially in those which are published and are intended to be used for later genotypephenotype correlations.

As all rearrangements were balanced according to cytogenetics and molecular cytogenetics and all patients were clinically normal (apart from infertility), no microarraybased comparative genomic hybridization (array CGH) analysis (13) of the breakpoints was deemed necessary (14). However, this type of analysis must be applied in cases of 


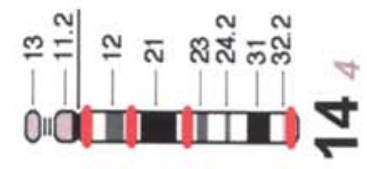

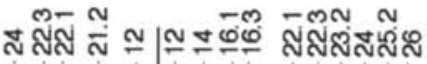
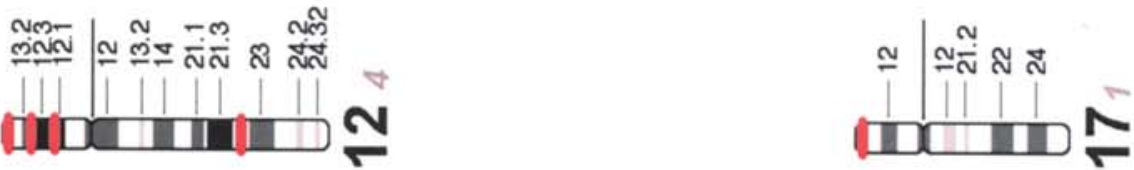

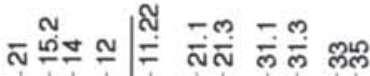

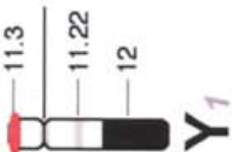

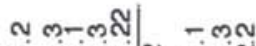

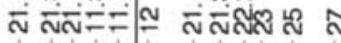
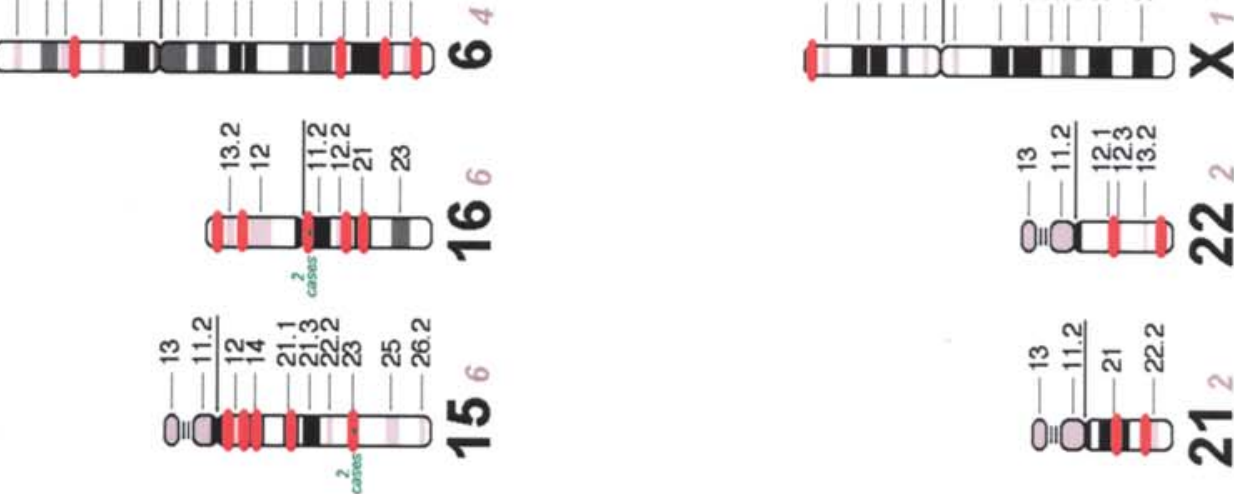

ํำ
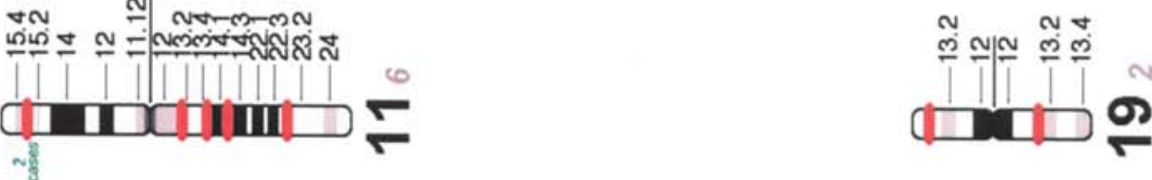

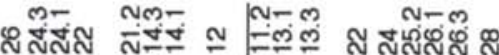
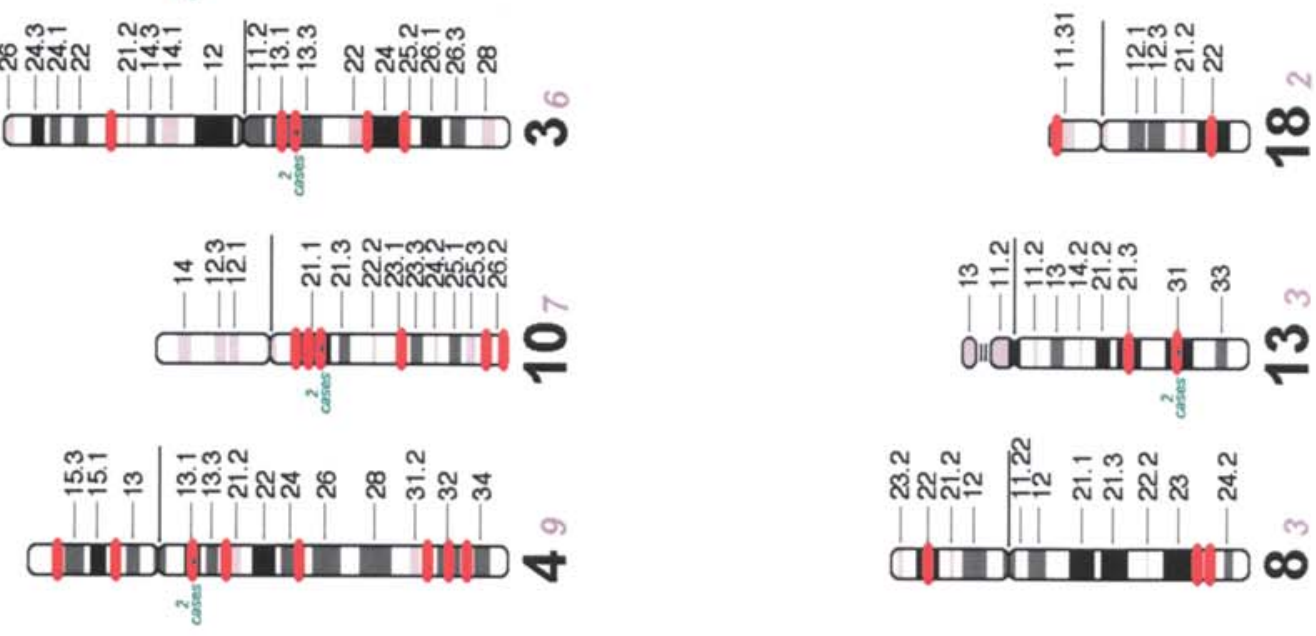

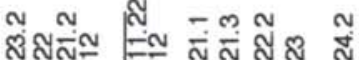

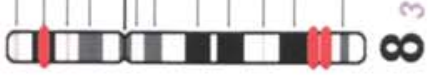

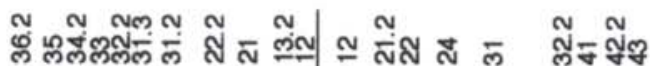

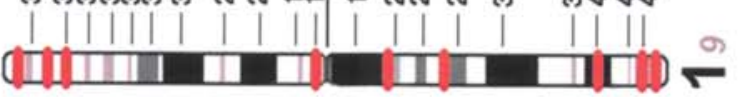

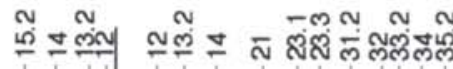
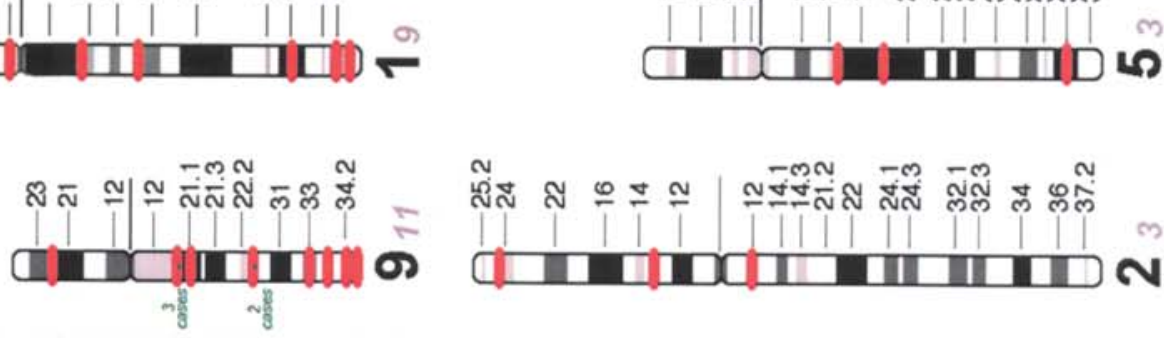

ผู่ส ส 
Table I. Listed are the 48 studied infertility cases. ${ }^{\mathrm{a}}$

\begin{tabular}{|c|c|c|c|}
\hline Case no. & Cytogenetic results & Molecular cytogenetic results & Applied FISH method \\
\hline \multicolumn{4}{|c|}{ Females with repeated abortions } \\
\hline 1 & $46, \mathrm{XX}, \mathrm{t}(1 ; 2)(\mathrm{p} 35 ; \mathrm{p} 14)$ & $\mathrm{t}(1 ; 2)(\mathrm{p} 36.1 ; \mathrm{p} 13)$ & MCB 1,2 \\
\hline 2 & $46, \mathrm{XX}, \mathrm{t}(1 ; 10)(\mathrm{q} 23.1 ; \mathrm{q} 11.2)$ & $\mathrm{t}(1 ; 10)(\mathrm{q} 23.3 ; \mathrm{q} 11.23)$ & MCB 1,$10 ;$ subcenM 10 \\
\hline 3 & $46, \mathrm{XX}, \mathrm{t}(2 ; 6)(\mathrm{p} 24.2 ; \mathrm{q} 25.1)$ & $\mathrm{t}(2 ; 6)(\mathrm{p} 24.2 ; \mathrm{q} 25.1)$ & MCB 2, 6 \\
\hline 4 & $46, X X, t(3 ; 6)$ & $\mathrm{t}(3 ; 6)(\mathrm{q} 13.2 ; \mathrm{q} 23)$ & $\operatorname{MCB} 3,6$ \\
\hline 5 & $46, X X, t(3 ; 9)$ & $\mathrm{t}(3 ; 9)(\mathrm{q} 25.1 ; \mathrm{p} 22)$ & MCB 3, 9; subtel 9p \\
\hline 6 & $46, \mathrm{XX}, \mathrm{t}(3 ; 19)(\mathrm{p} 21.3 ; \mathrm{q} 13.1)$ & $\mathrm{t}(3 ; 19)(\mathrm{p} 21.3 ; \mathrm{q} 13.13)$ & MCB 3, 19 \\
\hline 7 & 46,XX,t(4;9)(q35;q22) & $\mathrm{t}(4 ; 9)(\mathrm{q} 33 ; \mathrm{q} 13)$ & $\mathrm{MCB} 4,9$ \\
\hline 8 & 46,XX,t(4;10)(q12;q22.3) & $\mathrm{t}(4 ; 10)(\mathrm{q} 13.1 ; \mathrm{q} 23.1)$ & MCB 4, 10; subcenM 4 \\
\hline 9 & $46, X X, t(4 ; 22)(q 21.2 ; q 11.2)$ & $\mathrm{t}(4 ; 22)(\mathrm{q} 32 ; \mathrm{q} 12)$ & MCB 4; subcenM 22 \\
\hline 10 & $46, X X, t(5 ; 7 ; 12)$ & $\mathrm{t}(5 ; 7 ; 12)(\mathrm{q} 34 ; \mathrm{q} 11.21 ; \mathrm{p} 12.2)$ & MCB 5, 7, 12 \\
\hline 11 & $46, \mathrm{XX}, \mathrm{t}(5 ; 8)$ & $\mathrm{t}(5 ; 8)(\mathrm{q} 15 ; \mathrm{q} 23 \sim 24.1)$ & MCB 5, 8 \\
\hline 12 & 46,XX,t(5;16)(q13;p13.1 13.3) & $\mathrm{t}(5 ; 16)(\mathrm{q} 13.3 ; \mathrm{p} 13.3)$ & MCB 5, 16; subtel 16p \\
\hline 13 & 46,XX,t(7;9)(p13;q22) & $\mathrm{t}(7 ; 9)(\mathrm{p} 13 ; \mathrm{q} 22.3)$ & $\operatorname{MCB} 7,9$ \\
\hline 14 & $46, \mathrm{XX}, \mathrm{t}(7 ; 14)(\mathrm{q} 11.23 ; \mathrm{q} 22)$ & $\mathrm{t}(7 ; 14)(\mathrm{q} 11.23 ; \mathrm{q} 22)$ & $\operatorname{MCB} 7,14$ \\
\hline 15 & $46, \mathrm{XX}, \mathrm{t}(8 ; 10)(\mathrm{p} 23 ; \mathrm{q} 22.1)$ & $\mathrm{t}(8 ; 10)(\mathrm{p} 22 ; \mathrm{q} 21.2)$ & MCB 8, 10 \\
\hline 16 & $46, \mathrm{XX}, \mathrm{t}(9 ; 15)(\mathrm{q} 21 ; \mathrm{q} 21)$ & $\mathrm{t}(9 ; 15)(\mathrm{q} 13 ; \mathrm{q} 21.1)$ & MCB 9, 15; subcenM 9 \\
\hline 17 & 46,XX,t(9;16)(q34;p13.1) & $\mathrm{t}(9 ; 16)(\mathrm{q} 34.3 ; \mathrm{p} 13.1)$ & MCB 9, 16 \\
\hline 18 & 46,XX,t(11;15)(p15;q25) & $\mathrm{t}(11 ; 15)(\mathrm{p} 15.3 ; \mathrm{q} 23)$ & MCB 11,15 \\
\hline 19 & $46, X X, t(11 ; 15)(q 13.5 ; q 13)$ & $\mathrm{t}(11 ; 15)(\mathrm{q} 13.1 ; \mathrm{q} 14)$ & MCB 11,15 \\
\hline 20 & 46,XX,t(11;18)(q22;q22) & $\mathrm{t}(11 ; 18)(\mathrm{q} 14.2 ; \mathrm{q} 22)$ & MCB 11,18 \\
\hline
\end{tabular}

Partner of female with repeated abortions

$21 \quad 46, \mathrm{XY}, \mathrm{t}(2 ; 14) \quad \mathrm{t}(2 ; 14)(\mathrm{q} 12 ; \mathrm{q} 32.33)$

MCB 2, 14; subcenM 2; subtel $14 \mathrm{q}$

Aberration detected in pregnancy with fetus carrying an imbalance

$\begin{array}{lll}22 & 46, X X, \text { ins }(1 ; 3)(\mathrm{q} 44 ; \mathrm{q} 21 \mathrm{q} 25) & \operatorname{ins}(1 ; 3)(\mathrm{q} 43 ; \mathrm{q} 13.2 \mathrm{q} 23) \\ 23 & 46, \mathrm{XX}, \mathrm{t}(1 ; 4)(\mathrm{p} 36.3 ; \mathrm{q} 31.3) & \mathrm{t}(1 ; 4)(\mathrm{p} 36.3 ; \mathrm{q} 31.3) \\ 24 & 46, \mathrm{XY}, \mathrm{t}(12 ; 20)(\mathrm{q} 22 ; \mathrm{p} 11.2) & \mathrm{t}(12 ; 20)(\mathrm{q} 22 ; \mathrm{p} 13) \\ & & \\ 25 & 46, \mathrm{XY}, \text { ins }(15 ; 13)(\mathrm{q} 13 ; \mathrm{q} 32 \mathrm{q} 34) & \operatorname{ins}(15 ; 13)(\mathrm{q} 13 ; \mathrm{q} 31.2 \mathrm{q} 21.3)\end{array}$

MCB 1, 3

MCB 1, 4; subtel 1p MCB 12; subcenM 20; subtel 20p

MCB 13, 15;

LSI UBE3A; LSI PML; Subtel $13 q$

Aberration detected in parent of abnormal child carrying an imbalance

$\begin{array}{lll}26 & 46, \mathrm{XY}, \mathrm{t}(1 ; 17) & \mathrm{t}(1 ; 17)(\mathrm{q} 44 ; \mathrm{p} 13.2) \\ 27 & 46, \mathrm{XX}, \mathrm{t}(6 ; 22) & \mathrm{t}(6 ; 22)(\mathrm{q} 26 \sim \mathrm{q} 27 ; \mathrm{q} 13.32) \\ 28 & 46, \mathrm{XX}, \mathrm{t}(9 ; 14) & \mathrm{t}(9 ; 14)(\mathrm{q} 21.1 ; \mathrm{q} 11.1) \\ 29 & 46, \mathrm{XX}, \mathrm{t}(10 ; 13) & \mathrm{t}(10 ; 13)(\mathrm{q} 26.3 ; \mathrm{q} 31.2)\end{array}$

Unexplained infertility in partnership

46,X,der(X)t(X;11)(Xqter->Xp22.32 22.33: :11q13.5->11qter), der(11)t(Y;?X;11)(Ypter$>$ Yp11.3::?Xp22.32 22.33->?Xp22.33: :11q13.5->11pter)[65]/45,der(X)t(X;11) (Xqter->Xp22.32 22.33::11q13.5-

$>11$ qter), der(11)t(Y;?X;11)(Ypter->Yp11.3: :?Xp22.32 22.33->?Xp22.33:

$: 11 \mathrm{q} 13.5->11 \mathrm{pter})[5]$
MCB 1, 17; subtel 1q, 17p MCB 6; subtel 6q, 22q MCB 9, 14; subcenM 9, 14 MCB 10, 13; subtel 10q

MCB X, 11; subcenM 11; LSI KAL; LSI SRY; subtel Xp Yp 
Table I. Continued.

\begin{tabular}{|c|c|c|c|}
\hline Case no. & Cytogenetic results & Molecular cytogenetic results & Applied FISH method \\
\hline 32 & $46, \mathrm{XX}, \mathrm{t}(1 ; 7)(\mathrm{q} 24.1 ; \mathrm{q} 11.23)$ & $\mathrm{t}(1 ; 7)(\mathrm{q} 41 ; \mathrm{q} 11.22)$ & MCB 1, 7; LSI ELN; subcenM 7 \\
\hline 33 & $46, \mathrm{XX}, \mathrm{t}(1 ; 9)$ & $\mathrm{t}(1 ; 9)(\mathrm{p} 35 ; \mathrm{q} 22.3)$ & MCB 1,9 \\
\hline 34 & $46, \mathrm{XX}, \mathrm{t}(3 ; 4)(\mathrm{q} 12 ; \mathrm{q} 12)$ & $\mathrm{t}(3 ; 4)(\mathrm{q} 13.1 ; \mathrm{q} 21.1)$ & MCB 3, 4; subcenM 3 \\
\hline 35 & $46, X X, \operatorname{der}(4)(q)$ & $\operatorname{inv}(4)(q 13.1 q 25)$ & MCB 4 \\
\hline 36 & $46, \mathrm{XY}, \mathrm{t}(4 ; 16)(\mathrm{p} 16.1 ; \mathrm{p} 11.2)$ & $\mathrm{t}(4 ; 16)(\mathrm{p} 16.1 ; \mathrm{q} 11.1)$ & MCB 4; subcenM 16 \\
\hline 37 & 46,XY,t(4;21)(p14;q22.1) & $\mathrm{t}(4 ; 21)(\mathrm{p} 14 ; \mathrm{q} 22.1)$ & $\operatorname{MCB} 4,21$ \\
\hline 38 & $46, \mathrm{XX}, \mathrm{t}(6 ; 19)(\mathrm{p} 12.1 ; \mathrm{p} 12)$ & $\mathrm{t}(6 ; 19)(\mathrm{p} 21.3 ; \mathrm{p} 13.3)$ & MCB 6, 19; subcenM 19 \\
\hline 39 & $46, \mathrm{XY}, \mathrm{t}(8 ; 12)$ & $\mathrm{t}(8 ; 12)(\mathrm{q} 24.1 ; \mathrm{p} 13.1)$ & MCB 8, 12 \\
\hline 40 & $46, \mathrm{XX}, \operatorname{der}(9)(\mathrm{q})$ & $\operatorname{inv}(9)(\mathrm{q} 33 \mathrm{q} 34.2)$ & MCB 9; LSI bcr/abl; subtel 9q \\
\hline 41 & $46, \mathrm{XX}, \operatorname{der}(9)$ & $\operatorname{inv}(9)(q 13 q 34.1)$ & MCB 9 \\
\hline 42 & $46, X X, \operatorname{inv}(10)$ & $\operatorname{inv}(10)(\mathrm{q} 21.1 \mathrm{q} 26.1)$ & MCB 10 \\
\hline 43 & $46, \mathrm{XX}, \mathrm{t}(10 ; 16)(\mathrm{q} 11.2 ; \mathrm{q} 12.1)$ & $\mathrm{t}(10 ; 16)(\mathrm{q} 21.2 ; \mathrm{q} 12.2 \sim 13)$ & MCB 10,$16 ;$ subcenM 10, 16 \\
\hline 44 & 46,XY,t(11;15)(p15;p13) & $\mathrm{t}(11 ; 15)(\mathrm{p} 15.3 ; \mathrm{p} 11.2)$ & MCB 11,15 \\
\hline 45 & $46, X Y, t(11 ; 18)(q 23.3 ; p 11.32)$ & $\mathrm{t}(11 ; 18)(\mathrm{q} 23.1 ; \mathrm{p} 11.32)$ & MCB 11,$18 ;$ subtel 11q, 18p \\
\hline 46 & $46, X Y, t(12 ; 15)(p 13.3 ; q 24)$ & $\mathrm{t}(12 ; 15)(\mathrm{p} 13.3 ; \mathrm{q} 23)$ & MCB $12,15 / 3$ \\
\hline 47 & $46, X Y, t(14 ; 21)(q 22 ; q 22.1)$ & $\mathrm{t}(14 ; 21)(\mathrm{q} 13 ; \mathrm{q} 21)$ & MCB 14,$21 ;$ subcenM 14, 21 \\
\hline 48 & $46, X X, \operatorname{inv}(16)(q 11.2 q 13)$ & $\operatorname{inv}(16)(\mathrm{q} 11.1 \mathrm{q} 21)$ & MCB 16; subcenM 16 \\
\hline
\end{tabular}

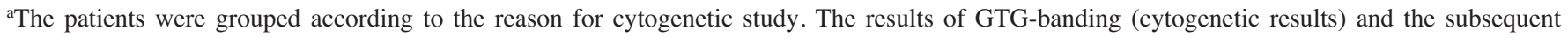
application of molecular cytogenetics are listed together with the applied FISH methods. abl, abl-oncogene in 9q34; bcr, breakpoint cluster region in 22q11.2; ELN, elastin gene in 7q11.2; FISH, fluorescence in situ hybridization; KAL, Kallmann syndrome 1 gene in Xp22.3; LSI, commercially available locus-specific probe (Abbott/Vysis); MCB, multicolor banding; PML, inducer of acute promyelocytic leukemia gene in 15q22; SRY, sexdetermining region Y in Yp11.3; subtel, commercially available subtelomeric probe (Abbott/Vysis); subcenM, subcentromere-specific multicolor FISH; and UBE3A, ubiquitin-protein ligase E3A in 15q13.

developmental delay and dysmorphic features and normal or apparently balanced karyotypes (15).

\section{Types of rearrangements observed}

Translocations. As expected the largest proportion of detected rearrangements in the studied 48 patients constituted cytogenetically balanced translocations; $83 \%$ of the cases had such a kind of rearrangement. In the majority of them only two chromosomes were involved, while in 2/40 balanced translocation cases three different chromosomes were contributing breakpoints. In the literature there are many reported cases on infertile couples in which one partner had such a rearrangement. However, similar to the aforementioned and discussed discordance of GTG-banding and molecular cytogenetic results confronted within this study there are few comparable cases available in the literature. What can be deduced from the literature is, that complex chromosomal rearrangements as in cases 10 and 30 are relatively rare events in infertility cases and can involve up to 5 or more different chromosomes (16-17). The most complex rearrangement observed in this study was case 30 involving chromosome X, Y and 11. The SRY-region was translocated here to the der(11); no similar cytogenetic case of an ' $\mathrm{XX}$ male' has been previously reported.

Insertions. Similar to complex chromosomal rearrangements, insertions of chromosomal material into another chromosome are extremely rare events. Here two such cases were found among the reported 48. To the best of our knowledge only three cytogenetically comparable cases with balanced insertion and infertility problems have been previously reported (18-20).

Inversions. In concordance with the literature, inversions were seen more frequently in connection with infertility; 6/48 cases $(12.5 \%)$ had an inversion. Apart from the frequently reported pericentric inversions of chromosomes 2 and 9 in connection with infertility (8) other unique inversions were reported in $\sim 22 \%$ of these cases (20).

Distribution of breakpoints in GTG-light and-dark bands. As summarized in Fig. 1 the 48 reported cases exhibited 100 breaks in 90 different chromosomal regions. In Table II the detected breakpoints were aligned with their localization within GTG-dark and GTG-light bands. Breakpoints detected in infertile patients with chromosomal rearrangements (Tables I and II) were prone to be in genetically more or less irrelevant regions; otherwise more severe clinical problems would have had to be expected in the rearrangement carriers. In discordance with this idea, 57/90 described breakpoints were located in GTG-light bands, which are known to be relatively gene-rich compared to the GTG-dark bands (21). Three out of ninety breaks appeared in the gene-poor hemiheterochromatic centromere-near regions of chromosomes 1 , 14 or 16 . Of the remaining observed breakpoints four were localized in the transient area of a GTG-dark to a GTG-light 
Table II. The molecular cytogenetic breakpoints from Table I are listed according to their chromosomal origin. ${ }^{\mathrm{a}}$

\begin{tabular}{|c|c|c|c|c|c|c|}
\hline \multirow[t]{2}{*}{ Breakpoint } & \multirow{2}{*}{$\begin{array}{l}\text { GTG-light } \\
\text { band }\end{array}$} & \multirow[t]{2}{*}{ Fragile sites } & \multirow{2}{*}{$\begin{array}{c}\text { Mariner } \\
\text { transposon-like } \\
\text { elements }\end{array}$} & \multirow{2}{*}{$\begin{array}{c}\text { Intrachromosomal } \\
\text { telomeric-like } \\
\text { sequences }\end{array}$} & \multicolumn{2}{|c|}{ Involvement } \\
\hline & & & & & Cancer & Evolution \\
\hline $\mathrm{Xp} 22.32 \sim 22.33$ & $\operatorname{mix}$ & $+\mathrm{Xp} 22.3^{\mathrm{b}}(30)$ & $+\mathrm{Xp} 22^{\mathrm{b}}(22)$ & & $+\mathrm{Xp} 22.3^{\mathrm{b}}(35)$ & \\
\hline Yp11.3 & $-\operatorname{mix}$ & & & & & \\
\hline $1 \mathrm{p} 36.3$ & + & $+1 \mathrm{p} 36^{\mathrm{b}}(23,28,30)$ & $+1 \mathrm{p} 36^{\mathrm{b}}(22)$ & $+1 \mathrm{p} 36^{\mathrm{b}}(23)$ & $+(33)$ & $+(33)$ \\
\hline $1 \mathrm{p} 36.1$ & + & $+1 \mathrm{p} 36^{\mathrm{b}}(23,28,30)$ & & $+1 \mathrm{p} 36^{\mathrm{b}}(23)$ & & $+(33)$ \\
\hline $1 \mathrm{p} 35$ & - & & $+(22)$ & $+(23)$ & & \\
\hline $1 \mathrm{p} 12$ & -(hemihet) & & & & & \\
\hline $1 \mathrm{q} 21.1$ & + & $+1 \mathrm{q} 21^{\mathrm{b}}(23,28,30)$ & & $+1 \mathrm{q} 21^{\mathrm{b}}(23)$ & $+(42)$ & $+(41)$ \\
\hline $1 \mathrm{q} 23.3$ & + & & & $+1 \mathrm{q} 23^{\mathrm{b}}(23)$ & $+(33)$ & $+(33)$ \\
\hline $1 q 41$ & - & & & & & \\
\hline $1 \mathrm{q} 43$ & - & & & & & \\
\hline $1 \mathrm{q} 44$ & + & $+(23,28,30)$ & & & $+(43)$ & $+(33)$ \\
\hline $2 \mathrm{p} 24.2$ & + & $+2 \mathrm{p} 24^{\mathrm{b}}(23,28,30)$ & & & & $+(41)$ \\
\hline $2 \mathrm{p} 13$ & + & $+(23,28,30)$ & & $+(23)$ & $+(33)$ & $+(33)$ \\
\hline $2 q 12$ & $-\operatorname{mix}$ & & $+(22)$ & & & \\
\hline $3 p 21.3$ & + & $+3 \mathrm{p} 21^{\mathrm{b}}(28)$ & $+3 \mathrm{p} 21^{\mathrm{b}}(22)$ & & $+(44)$ & \\
\hline $3 q 13.1$ & $-\operatorname{mix}$ & $+3 \mathrm{q} 13^{\mathrm{b}}(28)$ & $+3 \mathrm{q} 13^{\mathrm{b}}(22)$ & & & \\
\hline $3 q 13.2\{2 x\}$ & + & $+3 \mathrm{q} 13^{\mathrm{b}}(28)$ & $+3 \mathrm{q} 13^{\mathrm{b}}(22)$ & & & \\
\hline $3 q 23$ & + & & & & & \\
\hline $3 q 25.1$ & + & $+3 \mathrm{q} 25^{\mathrm{b}}(28,30)$ & & & & \\
\hline $4 \mathrm{p} 16.1$ & + & $+4 \mathrm{p} 16^{\mathrm{b}}(23,28,30)$ & $+4 \mathrm{p} 16^{\mathrm{b}}(22)$ & & & \\
\hline $4 \mathrm{p} 14$ & + & & & $+(23)$ & & \\
\hline $4 q 13.1\{2 x\}$ & $-\operatorname{mix}$ & & & $+4 \mathrm{q} 13^{\mathrm{b}}(23)$ & & \\
\hline $4 \mathrm{q} 21.1$ & + & $+4 \mathrm{q} 21^{\mathrm{b}}(28)$ & & $+4 \mathrm{q} 21^{\mathrm{b}}(23)$ & $+(33)$ & $+(33)$ \\
\hline $4 q 25$ & + & & & $+(23)$ & & \\
\hline $4 q 31.3$ & + & $+4 q 31^{b}(28)$ & & & & \\
\hline $4 q 32$ & $-\operatorname{mix}$ & & & $+(23)$ & & \\
\hline $4 q 33$ & + & & & & & \\
\hline $5 q 13.3$ & + & & & & & \\
\hline $5 q 15$ & + & $+(23,28,30)$ & & & & $+(41)$ \\
\hline $5 q 34$ & - & & $+(22)$ & & & \\
\hline $6 \mathrm{p} 21.3$ & + & & $+6 \mathrm{q} 21^{\mathrm{b}}(22)$ & & & \\
\hline $6 q 23$ & + & & & & & \\
\hline $6 q 25.1$ & + & & & & & \\
\hline $6 q 26 \sim q 27$ & $\operatorname{mix}$ & $+6 \mathrm{q} 26^{\mathrm{b}}(28,30)$ & $+(22)$ & & $+(26)$ & $+(41)$ \\
\hline $7 \mathrm{p} 13$ & + & $+(28,30)$ & & & & \\
\hline $7 q 11.21$ & + & $+7 \mathrm{q} 11^{\mathrm{b}}(28,30)$ & $+7 \mathrm{q} 11^{\mathrm{b}}(22)$ & & $+7 \mathrm{q} 11.2^{\mathrm{b}}(45)$ & $+7 \mathrm{q} 11.2^{\mathrm{b}}(41)$ \\
\hline $7 q 11.22$ & - & $+7 \mathrm{q} 11^{\mathrm{b}}(28,30)$ & $+7 \mathrm{q} 11^{\mathrm{b}}(22)$ & & $+7 \mathrm{q} 11.2^{\mathrm{b}}(45)$ & $+7 \mathrm{q} 11.2^{\mathrm{b}}(41)$ \\
\hline $7 q 11.23$ & + & $+7 \mathrm{q} 11^{\mathrm{b}}(28,30)$ & $+7 \mathrm{q} 11^{\mathrm{b}}(22)$ & & $+7 \mathrm{q} 11.2^{\mathrm{b}}(45)$ & $+7 \mathrm{q} 11.2^{\mathrm{b}}(41)$ \\
\hline $8 \mathrm{p} 22$ & - & & & & & \\
\hline $8 \mathrm{q} 23 \sim 24.1$ & $\operatorname{mix}$ & & $+8 \mathrm{q} 23^{\mathrm{b}}(22)$ & & & \\
\hline $8 \mathrm{q} 24.1$ & + & $+(28,30)$ & $+8 \mathrm{q} 24.1^{\mathrm{b}}(22,46)$ & & $+(46)$ & $+(41)$ \\
\hline 9 p22 & + & & & & & \\
\hline $9 q 13\{3 x\}$ & + & $+(23)$ & & & & \\
\hline $9 q 21.1$ & $-\operatorname{mix}$ & & & & & \\
\hline $9 q 22.3\{2 x\}$ & + & $+9 q 22^{b}(23,28,30)$ & $+9 q 22^{b}(22)$ & $+9 q 22^{b}(23)$ & & \\
\hline $9 q 33$ & $-\operatorname{mix}$ & & & & & \\
\hline $9 q 34.1$ & + & & & & & \\
\hline $9 q 34.2$ & - & & & & & \\
\hline $9 q 34.3$ & + & & & & $+(33)$ & $+(33)$ \\
\hline $10 q 11.23$ & + & & & & & $+10 \mathrm{q} 11.2^{\mathrm{b}}(41)$ \\
\hline $10 \mathrm{q} 21.1$ & - & $+10 \mathrm{q} 21^{\mathrm{b}}(23,28,30)$ & $+(22)$ & $+10 \mathrm{q} 21^{\mathrm{b}}(23)$ & $+10 \mathrm{q} 21^{\mathrm{b}}(47)$ & \\
\hline
\end{tabular}


Table II. Continued.

\begin{tabular}{|c|c|c|c|c|c|c|}
\hline \multirow[t]{2}{*}{ Breakpoint } & \multirow{2}{*}{$\begin{array}{l}\text { GTG-light } \\
\text { band }\end{array}$} & \multirow[t]{2}{*}{ Fragile sites } & \multirow{2}{*}{$\begin{array}{c}\text { Mariner } \\
\text { transposon-like } \\
\text { elements }\end{array}$} & \multirow{2}{*}{$\begin{array}{c}\text { Intrachromosomal } \\
\text { telomeric-like } \\
\text { sequences }\end{array}$} & \multicolumn{2}{|c|}{ Involvement } \\
\hline & & & & & Cancer & Evolution \\
\hline $10 \mathrm{q} 21.2\{2 \mathrm{x}\}$ & + & $+10 \mathrm{q} 21^{\mathrm{b}}(23,28,30)$ & & $+10 \mathrm{q} 21^{\mathrm{b}}(23)$ & & \\
\hline $10 \mathrm{q} 23.1$ & - & $+10 \mathrm{q} 23^{\mathrm{b}}(28)$ & & $+10 \mathrm{q} 23^{\mathrm{b}}(23)$ & $+10 \mathrm{q} 23^{\mathrm{b}}(48)$ & \\
\hline $10 \mathrm{q} 26.1$ & + & $+(30)$ & $+10 \mathrm{q} 26^{\mathrm{b}}(22)$ & & $+10 q 26^{b}(49)$ & $+(41)$ \\
\hline $10 \mathrm{q} 26.3$ & + & $+10 q 26^{b}(23,28,30)$ & & & & \\
\hline $11 \mathrm{p} 15.3\{2 \mathrm{x}\}$ & + & $+11 \mathrm{p} 15^{\mathrm{b}}(23,28)$ & +11 p15 pterb $(22)$ & & & \\
\hline $11 \mathrm{q} 13.1$ & + & $+11 \mathrm{q} 13^{\mathrm{b}}(23,28,30)$ & $+11 \mathrm{q} 13^{\mathrm{b}}(22)$ & $+11 \mathrm{q} 13^{\mathrm{b}}(23)$ & & $+11 \mathrm{q} 13^{\mathrm{b}}(41)$ \\
\hline $11 \mathrm{q} 13.5$ & + & $+11 \mathrm{q} 13^{\mathrm{b}}(23,28,30)$ & $+11 \mathrm{q} 13^{\mathrm{b}}(22)$ & $+11 \mathrm{q} 13^{\mathrm{b}}(23)$ & & $+11 \mathrm{q} 13^{\mathrm{b}}(41)$ \\
\hline $11 \mathrm{q} 14.2$ & + & $+(30)$ & & & & $+(41)$ \\
\hline $11 \mathrm{q} 23.1$ & + & $+11 \mathrm{q} 23^{\mathrm{b}}(23,28)$ & & $+11 \mathrm{q} 23^{\mathrm{b}}(23)$ & $+11 \mathrm{q} 23^{\mathrm{b}}(50)$ & \\
\hline $12 \mathrm{p} 13.3$ & + & & $+12 \mathrm{p} 13^{\mathrm{b}}(22)$ & & $+(33)$ & $+(33)$ \\
\hline $12 \mathrm{p} 13.1$ & + & & $+12 \mathrm{p} 13^{\mathrm{b}}(22)$ & & $+12 \mathrm{p} 13^{\mathrm{b}}(51)$ & \\
\hline $12 \mathrm{p} 12.2$ & + & & & & $+(33)$ & $+(33)$ \\
\hline $12 \mathrm{q} 22$ & + & & & & & \\
\hline $13 q 21.3$ & $-\operatorname{mix}$ & $+13 \mathrm{q} 21^{\mathrm{b}}(23,28)$ & & & & $+(33)$ \\
\hline $13 q 31.2\{2 x\}$ & + & & & & & \\
\hline $14 q 11.1$ & -(hemihet) & & & & & \\
\hline $14 q 13$ & + & & & & & \\
\hline $14 q 22$ & + & & & & & \\
\hline $14 q 32.33$ & + & & $+14 \mathrm{q} 32^{\mathrm{b}}(22)$ & & $+14 q 32^{b}(52)$ & \\
\hline $15 \mathrm{p} 11.2$ & + & & & & & \\
\hline $15 q 13$ & + & & & & & \\
\hline $15 q 14$ & - & & & & & \\
\hline $15 q 21.1$ & - & & & & & \\
\hline $15 \mathrm{q} 23\{2 \mathrm{x}\}$ & - & & & & & \\
\hline $16 \mathrm{p} 13.3$ & + & $+16 \mathrm{p} 13^{\mathrm{b}}(28)$ & $+16 \mathrm{p} 13.3^{\mathrm{b}}(22)$ & & & \\
\hline $16 \mathrm{p} 13.1$ & + & $+16 \mathrm{p} 13^{\mathrm{b}}(28)$ & $+16 \mathrm{p} 13.3^{\mathrm{b}}(22)$ & $+(23)$ & $+(33)$ & $+(33)$ \\
\hline $16 q 11.1\{2 x\}$ & -(hemihet) & $+(30)$ & & & & \\
\hline $16 q 12.2 \sim 13$ & $\operatorname{mix}$ & & & & & \\
\hline $16 q 21$ & - & & & & & \\
\hline $17 \mathrm{p} 13.2$ & - & & $+17 \mathrm{p} 13^{\mathrm{b}}(29)$ & & & \\
\hline $18 p 11.32$ & + & & & & & \\
\hline $18 \mathrm{q} 22$ & $-\operatorname{mix}$ & & $+(22)$ & & & \\
\hline $19 \mathrm{p} 13.3$ & + & & $+(22)$ & & & \\
\hline $19 q 13.13$ & + & $+19 \mathrm{q} 13.1^{\mathrm{b}}(28)$ & $+(22)$ & & & \\
\hline 20p13 & + & & & & & \\
\hline $21 \mathrm{q} 21$ & $-\operatorname{mix}$ & & $+21 \mathrm{q} 22^{\mathrm{b}}(22)$ & & & \\
\hline $21 \mathrm{q} 22.1$ & + & & & & & \\
\hline $22 q 12$ & $-\operatorname{mix}$ & $+(23,28)$ & & & $+(42)$ & $+(41)$ \\
\hline $22 \mathrm{q} 13.32$ & - & $+22 \mathrm{q} 13^{\mathrm{b}}(23,28)$ & & & & \\
\hline
\end{tabular}

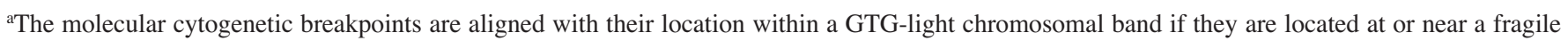
site, a Mariner transposon-like element or an intrachromosomal telomeric-like sequence and according to involvement of the corresponding region in cancer- or evolution-related breakpoints. The references are given in parentheses. hemihet, hemiheterochromatic band in centromeric or centromere-

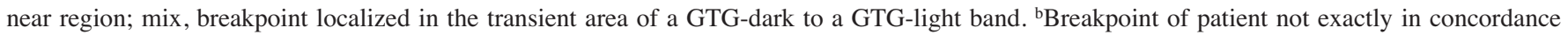
with fragile site, site of Mariner transposon-like element or intrachromosomal telomeric-like sequence; $\{2 \mathrm{x}\}$ or $\{3 \mathrm{x}\}$, same breakpoint detected 2 or 3 times in 48 patients.

band and twenty-six were located in GTG-dark bands. However, eleven of these last mentioned twenty-six breakpoints were located in GTG-dark bands known to split in GTG-light and -dark subbands. Because of this no clear conclusion could be drawn for these cases when the breakpoint was in a GTG-dark or -light subband. Thus, in summary only $11 / 90$ breakpoints $(\sim 12 \%)$ were undoubtfully located in GTG-dark bands, while the great majority were 
detected in the relatively gene-richer GTG-light bands of the karyotype. Thus, other reasons must be responsible for the observed breakpoint distribution.

Alignment of breakpoints with segmental duplications. Mariner transposon-like elements (MTLE) (22) and intrachromosomal telomeric-like sequences (ITS) (23) are special subgroups of the recently reported segmental duplications (24), which were shown to be involved in primate evolution and also in cancer development (25-27). Moreover, ITS and MTLE co-localize in parts with fragile sites (22-23,26,28-30). The latter are also reported to hold di-or tri-nucleotide repeats (31). In general, all segmental duplications such as ITS, MTLE and also fragile sites contribute to genomic instability (32). Here the characterized 90 chromosomal breakpoints detected in infertile but otherwise clinically normal patients were aligned with these aforementioned three elements (Table II). In $6 / 90$ breakpoints $(6.7 \%)$ a cytogenetic co-localization with ITS was observed, and in the additional 13, ITS were located near the observed breakpoints. In 9/90 (8.9\%) and 24/90 (26.9\%) of the cases the breaks were at or near the localization of MTLE, respectively. Finally, 10/90 (11.1\%) and 30/90 $(33.3 \%)$ of the break-events were at or near known fragile sites. In summary, $21 \%$ of the breakpoints in the studied 100 in infertility patients were near ITS; $35.8 \%$ co-localized with MTLE and $44.4 \%$ with fragile sites.

The relation of these sequences (fragile sites and segmental duplications) to cancer and evolution has been discussed among cytogeneticists (25-26,33-34). In Table II we summarized exemplary literature highlighting the fact that there appears to be a co-localization of fragile sites or other potentially unstable regions of the genome and breakpoints known from cancer or evolution cytogenetics.

The idea that these 'breakpoint-prone' regions could also be involved in chromosomal rearrangements in general and thus also in clinical genetics cases surprisingly is quite new (35-36). Raghavan and Lieber (36) suggest that some of the so-called non-B DNA structures, which are in part single stranded, are vulnerable to structure-specific nucleases and thus are the biological basis for constitutional chromosomal translocations or rearrangements in general.

Repeatedly observed breakpoints and segmental duplications/ fragile sites. Among the 90 breakpoints listed in Table II, 8 breakpoints were molecularly cytogenetically identical in two cases each and one breakpoint even in three cases. These breakpoints were co-localized with fragile sites (3q13.2, 9q13, 9q22.3, 10q21.2, 11p15.3, 16q11.1), ITS (4q13.1, 9q22.3, 10q21.2) and/or MTLE (3q13.2, 9q22.3, 11p15.3) in $77 \%$ of the cases (Table II). Only the breakpoints 13q31.2 and $15 q 23$ were not in correlation with any of these segmental duplication/fragile sites. Among the observed 81 breakpoints of the present study, 31 were not correlated with any segmental duplication/fragile sites (Table II). Thus, the correlation rate was $62 \%$ in 'single break-events' versus $77 \%$ in 'repeated break-events'.

Notably, the most frequently observed 'repeated breakevent' was present in 9q13. This band is known to be involved in constitutional pericentric inversions of chromosome 9, which occurs with a frequency of about $2 \%$ in the general population (37). Additionally, this region is known to be breakpoint prone and carries segmental duplications (38). Our own studies showed that the constitutional pericentric inversions of chromosome 9 are not due to one or two founder mutations but that they differ on a molecular level $(8,39$; our own unpublished data). Thus, an involvement of $9 \mathrm{p} 13$ and also 9p11 should be expected in cases as reported here. With high probability due to small sample size no 9 p11 breaks were described.

The presented data showed a strong positive correlation of breakpoints present in infertile patients with chromosome rearrangements and the location of fragile sites and of segmental duplications such as ITS and MTLE. These specific regions seem to be somewhat breakage prone and further studies are necessary to characterize the specific DNA-sequence base for this kind of feature.

In conclusion, evident from this molecular cytogenetic study of 48 infertility cases, out of a total of 90 involved, different chromosomal breakpoints confirmed a relatively high frequency of abnormal karyotypes found in couples with normovulatory women. This subgroup of patients seeking infertility treatment clearly benefits from a routine karyotype analysis as previously stated by Papanikolaou et al (40). Translocations, inversions, but also insertions are likely to be observed both in males and females of these couples. However, in our study these rearrangements were found at a gender-specific ratio of 1:3. Breaks are more likely to appear in GTG-light bands and in general in genomic regions prone to instability due to the presence of segmental duplications. However, further detailed molecular analysis will be necessary to study the mechanisms and genetic basis for this phenomenon.

\section{Acknowledgements}

This study was supported in part by the Evangelische Studienwerk e.V. Villigst, the Ernst-Abbe-Stiftung, the INTAS (AISbl 03-51-4060), the IZKF Jena (Start-up S16), IZKF together with the TMWFK (TP 3.7 and B307-04004) and the DFG (436 ARM 17/11/06, LI 820/9-1).

\section{References}

1. Shah K, Sivapalan G, Gibbons N, Tempest H and Griffin DK: The genetic basis of infertility. Reproduction 126: 13-25, 2003 .

2. Uehara S, Hashiyada M, Sato K, Sato Y, Fujimori K and Okamura K: Preferential X-chromosome inactivation in women with idiopathic recurrent pregnancy loss. Fertil Steril 76: 908-914, 2001.

3. Liehr T, Claussen U and Starke H: Small supernumerary marker chromosomes (sSMC) in humans. Cytogenet Genome Res 107: 55-67, 2004.

4. Daniely M, Aviram-Goldring A, Barkai G and Goldman B: Detection of chromosomal aberration in fetuses arising from recurrent spontaneous abortion by comparative genomic hybridization. Hum Reprod 13: 805-809, 1998.

5. Tharapel AT, Tharapel SA and Bannerman RM: Recurrent pregnancy losses and parental chromosome abnormalities: a review. Br J Obstet Gynaecol 92: 899-914, 1985.

6. Starke H, Seidel J, Henn W, Reichardt S, Volleth M, Stumm M, Behrend C, Sandig KR, Kelbova C, Senger G, Albrecht B, Hansmann I, Heller A, Claussen U and Liehr T: Homologous sequences at human chromosome 9 bands p12 and q13-21.1 are involved in different patterns of pericentric rearrangements. Eur J Hum Genet 10: 790-800, 2002. 
7. Liehr T, Heller A, Starke H, Rubtsov N, Trifonov V, Mrasek K, Weise A, Kuechler A and Claussen U: Microdissection based high resolution multicolor banding for all 24 human chromosomes. Int J Mol Med 9: 335-339, 2002.

8. Starke H, Nietzel A, Weise A, Heller A, Mrasek K, Belitz B, Kelbova C, Volleth M, Albrecht B, Mitulla B, Trappe R, Bartels I, Adolph S, Dufke A, Singer S, Stumm M, Wegner RD, Seidel J, Schmidt A, Kuechler A, Schreyer I, Claussen U, von Eggeling $\mathrm{F}$ and Liehr T: Small supernumerary marker chromosomes (SMCs): genotype-phenotype correlation and classification. Hum Genet 114: 51-67, 2003.

9. Liehr T, Heller A, Eichhorn KH, Beensen V, Schulze E, Starke H, Claussen U and Schreyer I: Inherited cryptic chromosomal aberrations may be more easily detected in their balanced forms: a case report with hidden $\operatorname{der}(1) \mathrm{t}(1 ; 17)(\mathrm{q} 44 ; \mathrm{p} 13.2)$. Prenat Diagn 24: 1022-1024, 2004.

10. Lemke J, Chudoba I, Senger G, Stumm M, Loncarevic IF Henry C, Zabel B and Claussen U: Improved definition of chromosomal breakpoints using high-resolution multicolour banding. Hum Genet 108: 478-483, 2001.

11. Heller A, Loncarevic IF, Glaser M, Gebhart E, Trautmann U, Claussen U and Liehr T: Breakpoint differentiation in chromosomal aberrations of hematological malignancies: Identification of 33 previously unrecorded breakpoints. Int $\mathrm{J}$ Oncol 24: 127-136, 2004.

12. Weise A, Starke H, Heller A, Tönnies H, Volleth M, Stumm M, Gabriele S, Nietzel A, Claussen U and Liehr T: Chromosome 2 aberrations in clinical cases characterised by high resolution multicolour banding and region-specific FISH probes. J Med Genet 39: 434-439, 2002.

13. Baptista J, Prigmore E, Gribble SM, Jacobs PA, Carter NP and Crolla JA: Molecular cytogenetic analyses of breakpoints in apparently balanced reciprocal translocations carried by phenotypically normal individuals. Eur J Hum Genet 13: 1205-1212, 2005.

14. Shaffer LG and Bejjani BA: Medical applications of array CGH and the transformation of clinical cytogenetics. Cytogenet Genome Res 115: 303-309, 2006.

15. Gribble SM, Prigmore E, Burford DC, Porter KM, Ng BL, Douglas EJ, Fiegler H, Carr P, Kalaitzopoulos D, Clegg S, Sandstrom R, Temple IK, Youings SA, Thomas NS, Dennis NR, Jacobs PA, Crolla JA and Carter NP: The complex nature of constitutional de novo apparently balanced translocations in patients presenting with abnormal phenotypes. J Med Genet 42: 8-16, 2005

16. Madan K, Nieuwint AW and van Bever Y: Recombination in a balanced complex translocation of a mother leading to a balanced reciprocal translocation in the child. Review of 60 cases of balanced complex translocations. Hum Genet 99: 806-815, 1997.

17. Kuechler A, Ziegler M, Blank C, Rommel B, Bullerdiek J, Ahrens J, Claussen U and Liehr T: A highly complex chromosomal rearrangement between five chromosomes in a healthy female diagnosed in preparation for intracytoplasmatic sperm injection. J Histochem Cytochem 53: 355-357, 2005.

18. Wang YT, Bajalica S, Han FY, Wang ZC, Bui TH and Xie YG: Direct and inverted reciprocal chromosome insertions between chromosomes 7 and 14 in a woman with recurrent miscarriages. Am J Med Genet 52: 349-351, 1994.

19. Starke H, Senger G, Kossakiewicz M, Tittelbach H, Rau D, Rubtsov N, Trifonov V, Heller A, Hartmann I, Claussen U and Liehr T: Maternal insertion of 18q11.2-q12.2 in 18p11.3 of the same chromosome analysed by microdissection and multicolour banding (MCB). Prenat Diagn 21: 1049-1052, 2001.

20. Rao L, Murthy K, Babu A, Venkata P, Deenadayal M and Singh L: Chromosome inversions and a novel chromosome insertion associated with recurrent miscarriages in South India. Arch Gynecol Obstet 272: 273-277, 2005.

21. Claussen U, Michel S, Mühlig P, Westermann M, Grummt UW, Kromeyer-Hauschild K and Liehr T: Demystifying chromosome preparation and the implications for the concept of chromosome condensation during mitosis. Cytogenet Genome Res 98: 136-146, 2002.

22. Reiter LT, Liehr T, Rautenstrauss B, Robertson HM and Lupski JR: Localization of mariner DNA transposons in the human genome by PRINS. Genome Res 9: 839-843, 1999.

23. Ruiz-Herrera A, Garcia F, Mora L, Egozcue J, Ponsa M and Garcia M: Evolutionary conserved chromosomal segments in the human karyotype are bounded by unstable chromosome bands. Cytogenet Genome Res 108: 161-174, 2005.
24. Bailey JA, Yavor AM, Massa HF, Trask BJ and Eichler EE: Segmental duplications: organization and impact within the current human genome project assembly. Genome Res 11: 1005-1017, 2001.

25. Sankoff D, Deneault M, Turbis P and Allen C: Chromosomal distributions of breakpoints in cancer, infertility, and evolution. Theor Popul Biol 61: 497-501, 2002.

26. Glover TW, Arlt MF, Casper AM and Durkin SG: Mechanisms of common fragile site instability. Hum Mol Genet 14: R197R205, 2005

27. Bailey JA and Eichler EE: Primate segmental duplications: crucibles of evolution, diversity and disease. Nat Rev Genet 7: 552-564, 2006.

28. Simonic I and Gericke GS: The enigma of common fragile sites. Hum Genet 97: 524-531, 1996.

29. Liehr T, Reiter LT, Lupski JR, Murakami T, Claussen U and Rautenstrauss B: Regional localization of 10 mariner transposonlike ESTs by means of FISH-evidence for a correlation with fragile sites. Mamm Genome 12: 326-328, 2001.

30. Denison SR, Simper RK and Greenbaum IF: How common are common fragile sites in humans: interindividual variation in the distribution of aphidicolin-induced fragile sites. Cytogenet Genome Res 101: 8-16, 2003.

31. Glover TW: Common fragile sites. Cancer Lett 232: 4-12, 2006.

32. Emanuel BS and Shaikh TH: Segmental duplications: an 'expanding' role in genomic instability and disease. Nat Rev Genet 2: 791-800, 2001

33. Miro R, Clemente IC, Fuster C and Egozcue J: Fragile sites, chromosome evolution, and human neoplasia. Hum Genet 75: 345-349, 1987.

34. Smith DI, Huang $H$ and Wang L: Common fragile sites and cancer (Review). Int J Oncol 12: 187-196, 1998.

35. Arlt MF, Durkin SG, Ragland RL and Glover TW: Common fragile sites as targets for chromosome rearrangements. DNA Repair. 5: 1126-1135, 2006.

36. Raghavan SC and Lieber MR: DNA structures at chromosomal translocation sites. Bioessays 28: 480-494, 2006.

37. Ait-Allah A, Ming P, Salem H and Reece E: The clinical importance of pericentric inversion of chromosome 9 in prenatal diagnosis. J Matern Fetal Invest 7: 126-128, 1979.

38. Humphray SJ, Oliver K, Hunt AR, Plumb RW, Loveland JE, Howe KL, Andrews TD, Searle S, Hunt SE, Scott CE, Jones MC, Ainscough R, Almeida JP, Ambrose KD, Ashwell RI, Babbage AK, Babbage S, Bagguley CL, Bailey J, Banerjee R, Barker DJ, Barlow KF, Bates K, Beasley H, Beasley O, Bird CP, Bray-Allen S, Brown AJ, Brown JY, Burford D, Burrill W, Burton J, Carder C, Carter NP, Chapman JC, Chen Y, Clarke G, Clark SY, Clee CM, Clegg S, Collier RE, Corby N, Crosier M, Cummings AT, Davies J, Dhami P, Dunn M, Dutta I, Dyer LW, Earthrowl ME, Faulkner L, Fleming CJ, Frankish A, Frankland JA, French L, Fricker DG, Garner P, Garnett J, Ghori J, Gilbert JG, Glison C, Grafham DV, Gribble S, Griffiths C, Griffiths-Jones S, Grocock R, Guy J, Hall RE, Hammond S, Harley JL, Harrison ES, Hart EA, Heath PD, Henderson CD, Hopkins BL, Howard PJ, Howden PJ, Huckle E, Johnson C, Johnson D, Joy AA, Kay M, Keenan S, Kershaw JK, Kimberley AM, King A, Knights A, Laird GK, Langford C, Lawlor S, Leongamornlert DA, Leversha M, Lloyd C, Lloyd DM, Lovell J, Martin S, Mashreghi-Mohammadi M, Matthews L, McLaren S, McLay KE, McMurray A, Milne S, Nickerson T, Nisbett J, Nordsiek G, Pearce AV, Peck AI, Porter KM, Pandian R, Pelan S, Phillimore B, Povey S, Ramsey Y, Rand V, Scharfe M, Sehra HK, Shownkeen R, Sims SK, Skuce CD, Smith M, Steward CA, Swarbreck D, Sycamore N, Tester J, Thorpe A, Tracey A, Tromans A, Thomas DW, Wall M, Wallis JM, West AP, Whitehead SL, Willey DL, Williams SA, Wilming L, Wray PW, Young L, Ashurst JL, Coulson A, Blocker H, Durbin R, Sulston JE, Hubbard T, Jackson MJ, Bentley DR, Beck S, Rogers J and Dunham I: DNA sequence and analysis of human chromosome 9. Nature 429: 369-374, 2004.

39. Schmidt S, Claussen U, Liehr T and Weise A: Evolution versus constitution: differences in chromosomal inversion. Hum Genet 117: 213-219, 2005.

40. Papanikolaou EG, Vernaeve V, Kolibianakis E, Assche EV, Bonduelle M, Liebaers I, Van Steirteghem A and Devroey P: Is chromosome analysis mandatory in the initial investigation of normovulatory women seeking infertility treatment? Hum Reprod 20: 2899-2903, 2005.

41. Newman TL, Tuzun E, Morrison VA, Hayden KE, Ventura M, McGrath SD, Rocchi M and Eichler EE: A genome-wide survey of structural variation between human and chimpanzee. Genome Res 15: 1344-1356, 2005. 
42. Egeli U, Ozkan L, Tunca B, Kahraman S, Cecener G, Ergul E and Engin $\mathrm{K}$ : The relationship between genetic susceptibility to head and neck cancer with the expression of common fragile sites. Head Neck 22: 591-598, 2000.

43. Jiahui X, Luyun L, Xiaoxuan H and Jianyun X: Fragile site $1 q 44$ involved in nasopharyngeal carcinoma. A study of a marker chromosome der(1)t $(1 ; 3)(\mathrm{q} 44 ; \mathrm{p} 11)$. Cancer Genet Cytogenet 35: 135-140, 1988.

44. Martinez A, Walker RA, Shaw JA, Dearing SJ, Maher ER and Latif F: Chromosome 3p allele loss in early invasive breast cancer: detailed mapping and association with clinicopathological features. Mol Pathol 54: 300-306, 2001.

45. Furuya T, Ochi H and Watanabe S: Common fragile sites in chromosomes of bone marrow cells and peripheral blood lymphocytes from healthy persons and leukemia patients. Cancer Genet Cytogenet 43: 131-138, 1989.

46. Ferber MJ, Thorland EC, Brink AA, Rapp AK, Phillips LA, McGovern R, Gostout BS, Cheung TH, Chung TK, Fu WY and Smith DI: Preferential integration of human papillomavirus type 18 near the c-myc locus in cervical carcinoma. Oncogene 22: 7233-7242, 2003.

47. Beder LB, Gunduz M, Ouchida M, Gunduz E, Sakai A, Fukushima K, Nagatsuka H, Ito S, Honjo N, Nishizaki K and Shimizu K: Identification of a candidate tumor suppressor gene RHOBTB 1 located at a novel allelic loss region 10q21 in head and neck cancer. J Cancer Res Clin Oncol 132: 19-27, 2006.
48. Ozisik YY, Meloni AM, Stone JF, Sandberg AA and Surti U: Spontaneous expression of the chromosome fragile site at 10q23 in leiomyoma. Cancer Genet Cytogenet 74: 73-75, 1994.

49. Nakagawachi T, Soejima H, Urano T, Zhao W, Higashimoto K, Satoh Y, Matsukura S, Kudo S, Kitajima Y, Harada H, Furukawa K, Matsuzaki H, Emi M, Nakabeppu Y, Miyazaki K, Sekiguchi $\mathrm{M}$ and Mukai T: Silencing effect of $\mathrm{CpG}$ island hypermethylation and histone modifications on O6-methylguanine-DNA methyltransferase (MGMT) gene expression in human cancer. Oncogene 22: 8835-8844, 2003.

50. Deveney R, Chervinsky DS, Jani-Sait SN, Grossi M and Aplan PD: Insertion of MLL sequences into chromosome band 5q31 results in an MLL-AF5Q31 fusion and is a rare but recurrent abnormality associated with infant leukemia. Genes Chromosomes Cancer 37: 326-331, 2003.

51. Raimondi SC, Shurtleff SA, Downing JR, Rubnitz J, Mathew S, Hancock M, Pui CH, Rivera GK, Grosveld GC and Behm FG: $12 \mathrm{p}$ abnormalities and the TEL gene (ETV6) in childhood acute lymphoblastic leukemia. Blood 90: 4559-4566, 1997.

52. Robinson HM, Taylor KE, Jalali GR, Cheung KL, Harrison CJ and Moorman AV: $\mathrm{t}(14 ; 19)(\mathrm{q} 32 ; \mathrm{q} 13)$ : a recurrent translocation in B-cell precursor acute lymphoblastic leukemia. Genes Chromosomes Cancer 39: 88-92, 2004. 